\title{
Dynamic Modeling and Parameter Identification of Magnetorheological Shock Absorber
}

\author{
Yang Yonggang, Yu Peng, Zhu Shixing and Kong Xiangfen \\ College of Aeronautical Engineering, Civil Aviation University of China, \\ Tianjin 300300, China \\ Yonggang_Yang@163.com
}

\begin{abstract}
By dynamic analysis, the dynamic modeling of designed magnetorheological(MR) shock absorber with metering pin is established, and using the experimental dato The relation of kinematic viscosity and current, and shear stress and curren are respectively identified, and the parameters of dynamic model are identified by the least square identification method. The experiment results show that the iden ified model has high accuracy, and can be used for the performance analysis and controlsystem design of the shock absorber.
\end{abstract}

Keywords: Magnetorheological shock absorber; Damping force model; Least square method; Parameters identification

\section{Introduction}

As a controlled fluid damper, MR damper has been successfully used in many areas, such as seismic resistance of bridge tutomobile shock absorber, etc. ${ }^{[1-5]}$. Because of the special characteristic of magnetorheological 1uid, it has a good prospect in terms of shock absorption. With the further research on MRF, its applications will continue to expand. When landing an taxiing of aircraft, a lot of impact energy will been produced and the shock absôrbers are need to dissipate the energy. although MRF damper on landing gear of aircrait has no practical application, there have been many experts and scholars were siudied in this regard. In China, Jia Yuhong research the possibility of magnetorheological damper on the landing gear applications, while designing the corresponding control Agorithm ${ }^{[6-8]}$, MR damper is presented for adaptive control of helicopter ground resonance by Wang Wei and Xia Pinqi ${ }^{[9]}$.Tian Jing et al. Simulate and Analyze semi-active control for MR damper landing gear ${ }^{[10]}$. In this paper, a new type of MR shock absorbers is presented, its dynamic model is studied, and some experiments are done to identify the dynamic model parameters using the least squares method. By comparison with the experimental results, the shock absorber damping force model has high accuracy, can be used to control the shock absorber.

\section{Structure of MR Shock Absorber}

To ensure reliability and damping force adjustable properties of shock absorber, based on conventional oleo-pneumatic shock absorber with metering pin, a shock absorber based on MR fluid with metering pin (called MR shock absorber) is designed, so that it's damper effect can be simultaneously controlled by current and stroke, then which will improve efficiency and damping force adjustment range while improve the safety factor of the shock absorber. Its structure diagram is shown in Figure 1.

In operating the MR shock absorber, piston rod drives the guide plate to press against the chamber, the MR fluid on the upper end of guide plate flows through the orifice on the top of piston rod, then through the upper portion hollow of the piston rod, finally 
through annular gap of piston by flowing through the radial orifice in the piston rod. The piston of the shock absorber is axially provided with a number of rectangular annular grooves. On the action of external electromagnetic field, the MR fluid flows through the rectangular grooves with the expansion of its volume and decrease of its velocity. Because of the dissipation effect of the rectangular grooves produced vortex, the kinetic energy is turned into heat energy, so MR shock absorber with rectangular annular groove has the effect of enhancing the damping force. In addition, by using tapered metering pin, damping force of the shock absorber varies with the stroke change, and when the semi-active control of MR damper is failure, the shock absorber can degenerate into an efficient oleo-pneumatic shock absorber, which makes landing gear shock absorber system more secure.

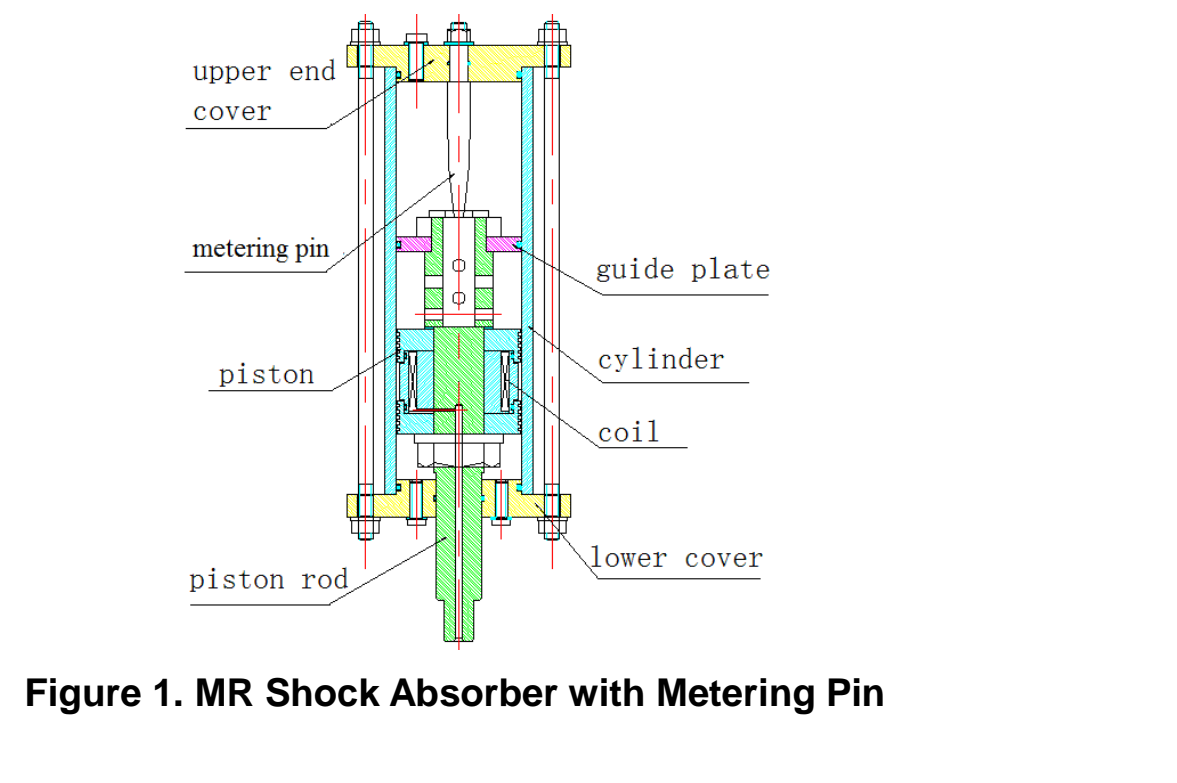

\section{Dynamic Model of MR Shock Absorber}

For getting a good effective of semi-active control by using MR shock absorbers shock, an aceurate dynamic model is one of the key factors. Taking the piston as the study object, $M$ expresses the quality of the piston. The differential equation can be obtained for

$$
\text { 2 } M \ddot{s}=F_{d}+F_{a}+F_{r}
$$

Where $s$ is displacement of the piston, $F_{d}$ is an external driving force applying on the piston rod, $F_{a}$ is gas elastic force, $F_{r}$ is the damper produced by MR shock absorber. In the compression stroke, $F_{d}$ and $F_{a}, F_{r}$ are opposite, where $F_{a}$ and $F_{a}, F$ are opposite in the stretch stroke. $F_{r}$ consists of the following components, viscous damping force $F_{\eta}$ in annular damp channel, Coulomb damping force $F_{m r}$ generated by MRF, MR fluid inertia force $F_{j}$ generated under high-speed impact, throttle resistance $F_{v}$, friction force $F_{f}$. So the total damping force equation can be expressed as:

$$
F_{r}=F_{\eta}+F_{m r}+F_{j}+F_{v}+F_{f}
$$




\subsection{Viscous Damping Force and Coulomb Damping Force}

When the MR damping fluid is squeezed into the damp channel, due to the viscosity of MRF, and the friction between the liquid and the wall and between the liquid and the liquid will generate the viscous damping force, named $F_{\eta}$. When subjected to different magnetic in damping channel, MRF will generate additional Coulomb damping force $F_{m r}$ because of MR effect. The value of $F_{\eta}$ and $F_{m r}$ relate to MRF viscosity, the magnetic field strength and the length of annular damping channel. Based on the mode of pressure difference or shear flow in the MR damping channel, the output of the viscous damping force and Coulomb damping force can be expressed as ${ }^{[11]}$

$$
F_{\eta}+F_{\mathrm{mr}}=\left(\frac{3 L A_{P}}{h}+L \pi D\right) \tau_{y} \operatorname{sgn}(v(t))+\left(\frac{12 \eta L A_{p}^{2}}{\pi D h^{3}}+\frac{L \pi D \eta}{h}\right) v(t)
$$

$A_{p}$ is the effective area of the piston under pressure, $v(t)$ is the relative speed of the piston and the cylinder, $D$ is the diameter of piston, $f_{c}$ is the length of damping channel, $\mathrm{h}$ is the work space, $\eta$ is the dynamic viscosity of the fluid and $\tau_{y}$ is the yield stress.

\subsection{Fluid Inertia Force}

MR fluid would be acted by inertia due to the mass and the acceleration of the liquid when MR fluid flows. This generated resisfance to the MR fluid is called inertial resistance and denoted by $F_{j}$. When the absorte value of the acceleration is very small, the inertial forces can be neglected. And when impathoad is added to the MR shock absorber, acceleration amplitude is large and the inertial force could not be ignored. According to the reference [12] the pertia of the fluid resistance can be expressed as:

$$
A=\frac{\operatorname{L\rho } A_{p}^{2}\left(A_{p}-A_{d}\right)}{A_{d}} \dot{v}(t)
$$

In which, $c_{1}$ is the are of the damping channel, $\rho$ is density of magnetic variation liquid, and $\dot{v}(t)$ is relative acceleration of the piston and cylinder. By the formula (4), when the MR damper structute is determined, the inertial force of the piston is only related to the-acceleration and is uncontrollable.

\subsection{Throttle Resistance}

Throttle resistance 18 ' generated from the sudden change of the cross-section of MR flow, which is proportional to the square of piston speed, and can be expressed as ${ }^{[12]}$ :

$$
F_{v}=\frac{\rho A_{p}^{3}}{2\left(A_{f} C_{d}\right)^{2}} v(t)^{2}
$$

\footnotetext{
A is the area of the hole, and changes with the relative displacement of the cylinder and the piston. And $C_{d}$ is flow coefficient of oil holes.
}

\subsection{Friction}

Due to mechanical friction damping force associated with the system, such as the sliding friction between the inner wall of the cylinder and guide head, the sliding friction between the piston and the seal block, and the frictions from the surrounding environment or related structures in the external dampers, etc. The sum of these frictions can be described as:

$$
F_{f}=f_{0} \operatorname{sign}(v(t))
$$


In which, $f_{0}$ represents the estimated value of friction, which is much smaller than friction in MR damper and has little effect on the damping force of the shock absorber. The total damping force can be expressed as shown in Equation (7):

$$
\begin{aligned}
F_{\mathrm{r}} & =\left(\frac{12 \eta L A_{p}^{2}}{\pi D h^{3}}+\frac{L \pi D \eta}{h}\right) v(t)+\left(\frac{3 L A_{P}}{h}+L \pi D\right) \tau_{y} \operatorname{sgn}(v(t)) \\
& +\frac{L \rho A_{p}^{2}\left(A_{p}-A_{d}\right)}{A_{d}} \dot{v}(t)+\frac{\rho A_{p}^{3}}{2\left(A_{f} C_{d}\right)^{2}} v(t)^{2}+f_{0} \operatorname{sign}(v(t))
\end{aligned}
$$

\subsection{Gas Elastic Force}

The variation of gas elastic force in buffer during landing buffer is described by the changing process model.

$$
F_{a}=A_{1} P_{0}\left(\frac{V_{0}}{V_{0}-A_{p} s}\right)^{n}-P_{s}\left(A_{1}-A_{p}\right)
$$

$A_{1}$ is the area of piston, $P_{0}$ is initial inflation pressure of buffer, $P_{s}$ is atmospheric pressure outside, $V_{0}$ is the initial volume of the gas chamber, and $n$ is polytropic exponent of gas, which is generally ranging between 1.1.4, and $n=13$ is adopted.

According to the designed damper, structure size is shown in Table 1. And $\tau_{y}$ and $\eta$ vary with the applied magnetic field strengh, which can be denoted by polynomials of applied current. From the MR fluid properties and tested results by factory, cubic polynomial is adopted to meet the requirements, which is shown in Equation (9) and (10).

$$
\left\{\begin{array}{l}
I=C_{11} I^{3}+C_{12} I+C_{13} I+C_{10} \\
=C_{21} I^{3}+C_{22} I^{3}+C_{23} I+C_{20}
\end{array}\right.
$$

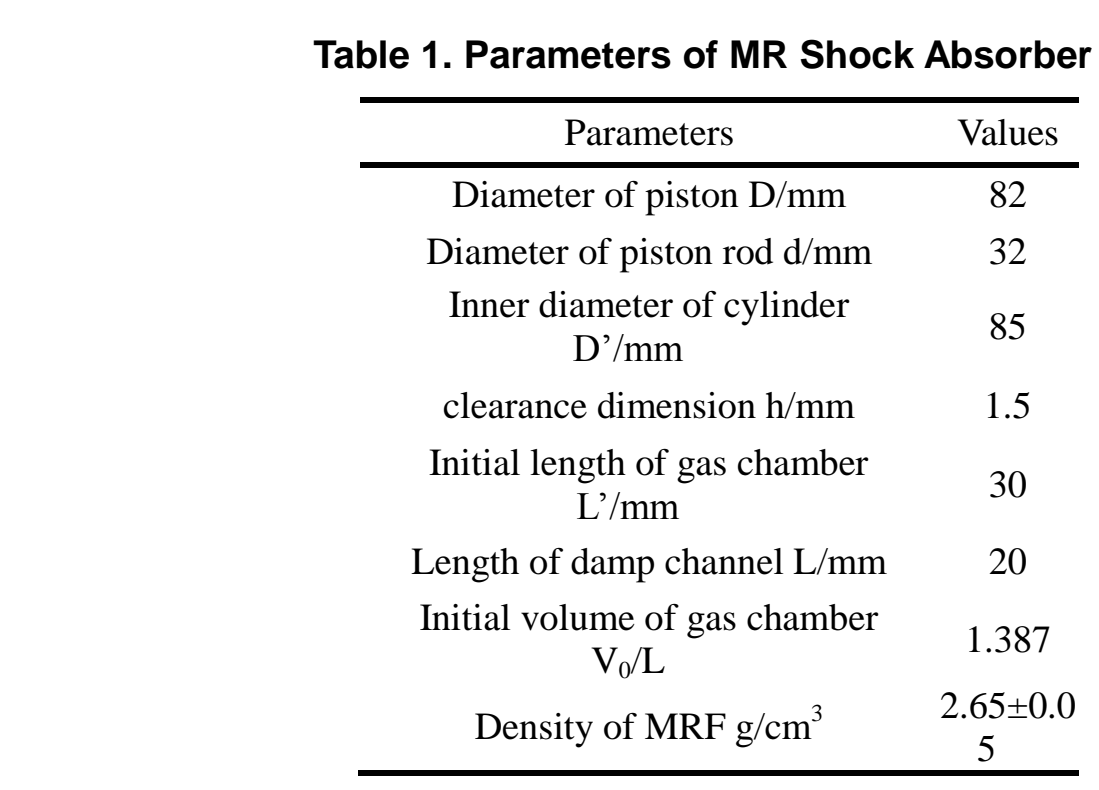

Taking equation (9) and (10) into the formula (7), the shock absorber damping force model can be obtained. Taking equation (7) and (8) into the formula (1), the kinetic model of this shock absorber can be obtained.

1.Model Parameter Identification and Analysis

4.1 Parameter Identification 
By dynamic analysis, all parameters and coefficients in dynamic model are known except from $C_{10}$ to $C_{23}$ and $f_{0}$. To identify the unknown parameter and coefficients, the loading vibration experiments are done with the current was $0 \mathrm{~A}, 0.1 \mathrm{~A}, 0.3 \mathrm{~A}, 0.5 \mathrm{~A}$, and frequency and current keep const. According to the experimental data, the least squares method is used to identify the parameters of each model, from which mechanical model of MR damping force is shown as:

$$
\begin{aligned}
F_{\mathrm{r}}= & 3.64 \times v(t) \times \eta+7.461 \times \operatorname{sgn}(v(t)) \times \tau_{y}+8.501 \times \dot{v(t)} \\
& +1.313 \times 10^{-4} \times v(t)|v(t)|+2.041 \operatorname{sgn}(v(t))
\end{aligned}
$$

Where, $\eta=71.58 \times I^{3}-36.08 \times I^{2}+4.36 \times I+0.3221$

$$
\tau_{y}=-1219 \times I^{3}+846.9 \times I^{2}-134 \times I+83.33
$$

\subsection{Identification Analysis}

The relations of each velocity-force and displacement-force, the black line represents the result get from experiment and red line gets from the identified model, are shown respectively in Figure 2 and Figure 3 when the amplitude is $3 \mathrm{~mm}$, frequency is $4 \mathrm{HZ}$ and current is $0 \mathrm{~A}$. In Figure 4 and Figure 5, the current is adjusted to $0.3 \mathrm{~A}$ and other conditions are not changed. As can be seen from the figure, when the velocity approaches zero, there was a great deviation of the damping force between from model and experiment, did not reflect the good hysteresis characteristios of velocity-force, but fit within other velocity range well.

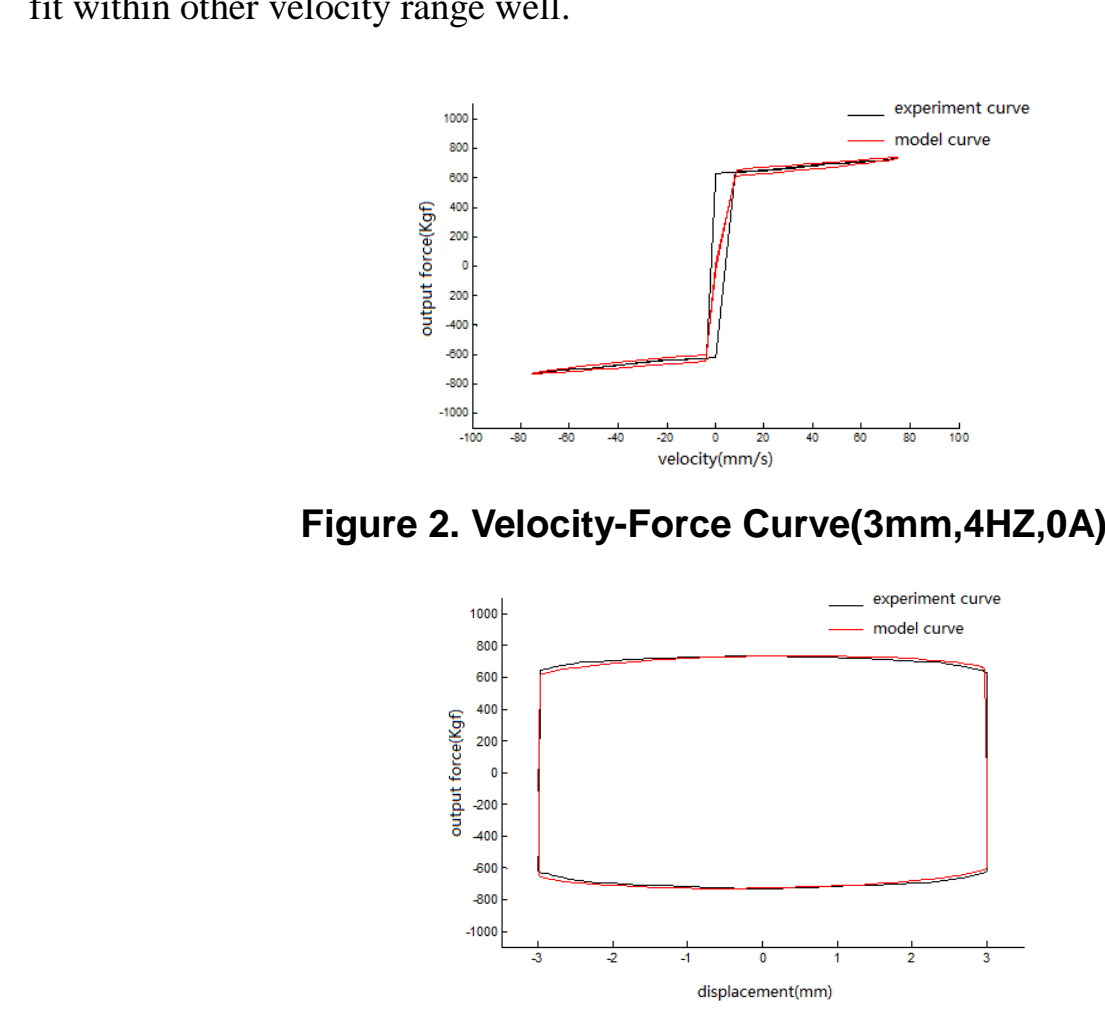

Figure 3. Displacement-Force Curve(3mm,4HZ,0A) 


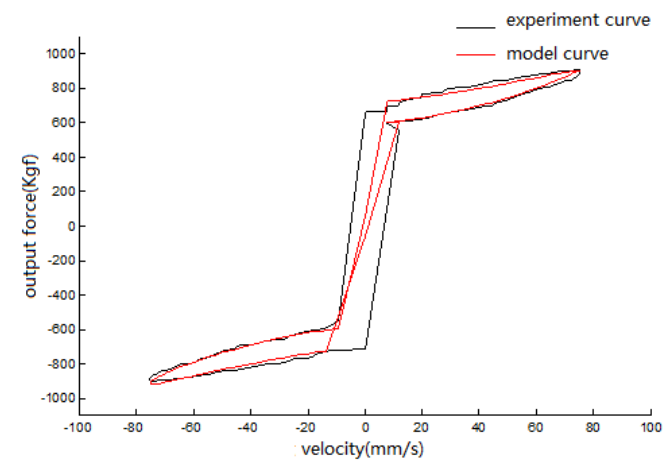

Figure 4. Velocity-Force Curve(3mm,4HZ, 0.3A)

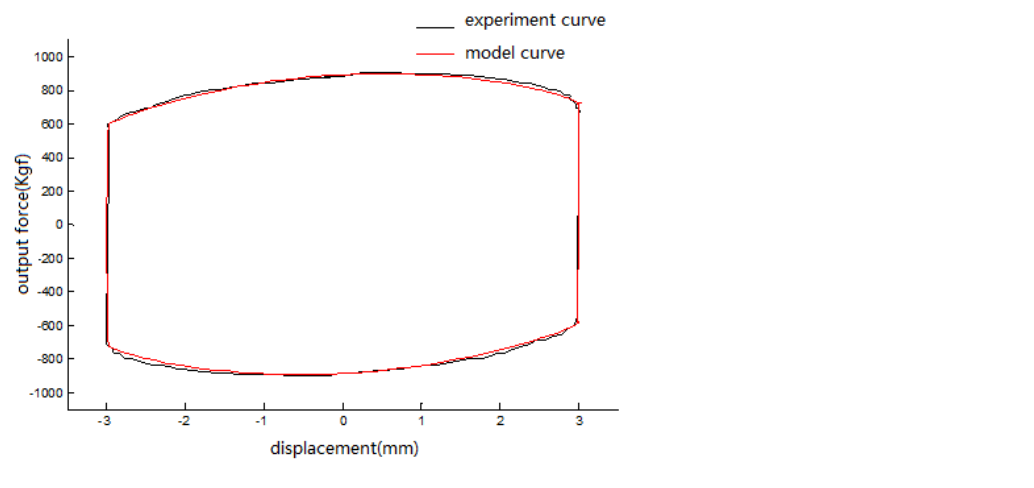

Figure 5. Displacement-Force Curve (3mm,4HZ, 0.3A)

\subsection{Inspection of Model}

To test the extensive of Dynamic model, the current and incentive are changed to examine the fitting degee between the outputs of the model and the outputs of experiment. Figure and Figure 7 are respectively velocity-force curve and displacement curve by changing cartent, the black lines get from experiment and red lines get from the theory model Fgure 8 is the output force error curve. Figure 9 and Figure 10 Figure 6 and Figane 7 are respectively velocity-force curve and displacement curve by changing current and Figure 11 is output force error curve.

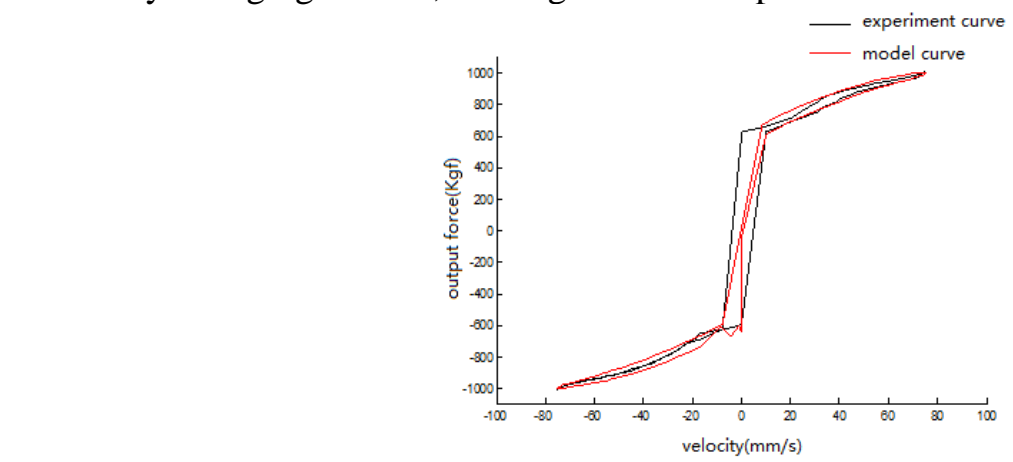

Figure 6. Velocity-Force Curve( $3 \mathrm{~mm}, 4 \mathrm{HZ}, 0.5 \mathrm{~A})$ 


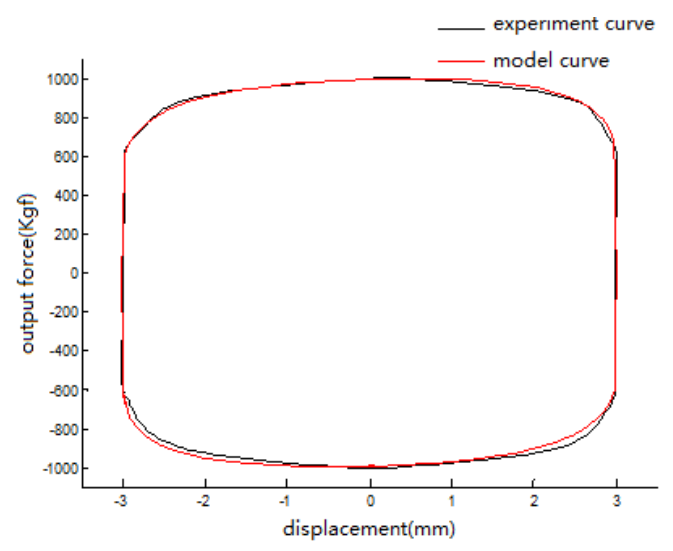

Figure 7. Displacement-Force Curve(3mm,4HZ, 0.5A)

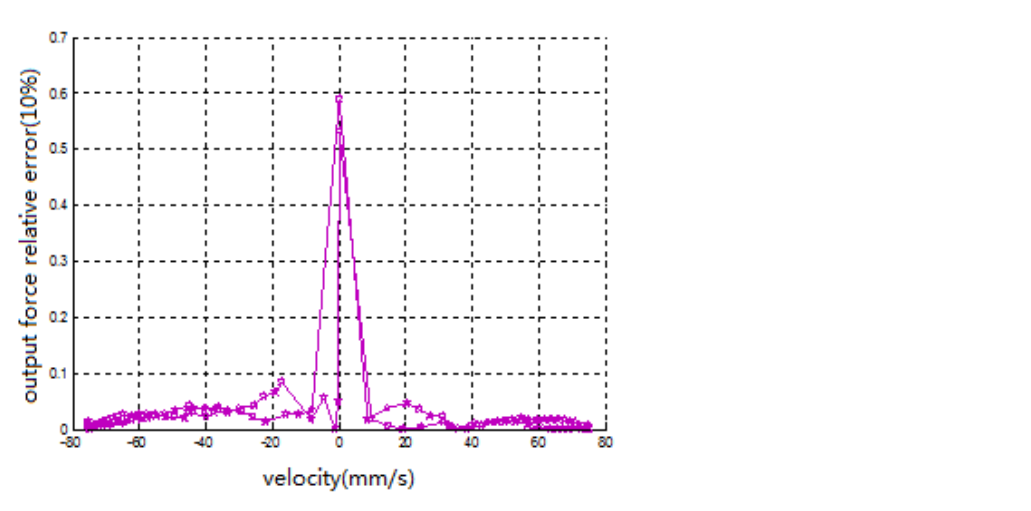

Figure 8. Velocity Force Error Curve(3mm,4HZ, 0.5A)

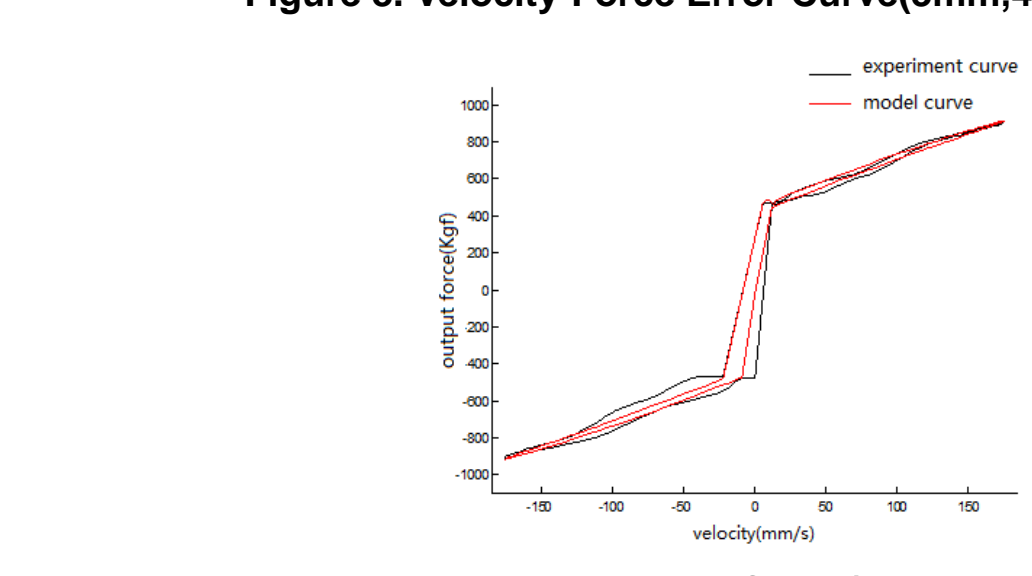

Figure 9. Velocity-Force Curve(7mm,4HZ, 0.3A) 


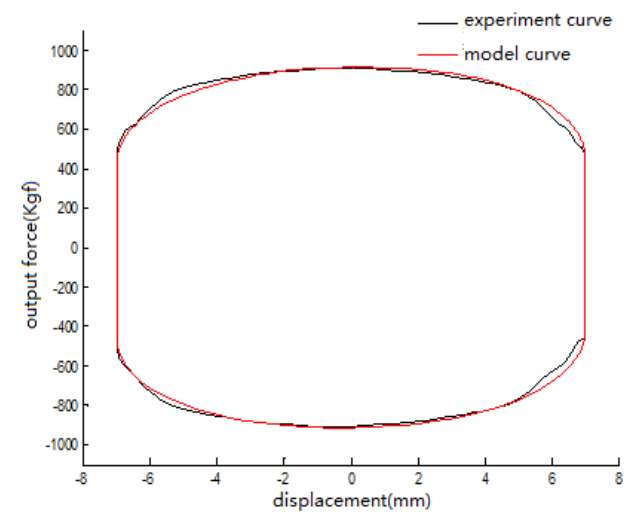

Figure 10. Displacement-Force Curve(7mm,4HZ, 0.3A)

Figure 11. Velocity Force Error Curve $(7 \mathrm{~mm}, 4 \mathrm{HZ}, 0.3 \mathrm{~A})$

The comparison results show the model having a very high fitting degree, which can better describe the change of MR shock absorber damping force versus its displacement. The maximum value of relative error about damping force versus speed is around $6 \%$ as shown in Figure 8and Figure 11, in an acceptable range.

\section{Conclusion}

Dynamic analysis and modeling of self-developed shock absorber are studied, and its properties were tested by bench, then the dynamic model parameters are identified based on the test data. By the way of changing the current and changing the displacement, the identified models were tested. The result shows that the model has a high fitting degree, which can be used to describe MR shock absorber damping force variation with different speed, and provide the foundation for controlling.

\section{Acknowledgments}

The authors would like to thank the anonymous referees for their valuable suggestions. The research work was supported by National Natural Science Foundation of China under Grant No. 61172013 and the Maor International Joint Research Program of China (Grant No. 2013DFA71120 and 2014DFB70120). 


\section{References}

[1] Y. T. Choi and N. M. Wereley, "Vibration Control of a Landing Gear System Featuring Electrorheological/Magnetorheological Fluids", Journal of Aircraft, (2003), vol. 40, no. 3, pp. 432-439.

[2] D. C. Batterbee, D. SimsN and R. Stanway, "Magnetorheological Landing Gear: 1. A Design Methodology", Smart Materials and Structures, vol. 16, no. 6, (2007), pp. 2429-2440.

[3] D. C. Batterbee, N. D. Sims and R. Stanway, "Magnetorheological Landing Gear: 2. Validation Using Experimental Data", Smart Materials and Structures, vol. 16, no. 6, (2007), pp. 2441-2452.

[4] T.-M. Zhou, E.-W. Chen and Y.-M. Lu, "Modified polynomial model and its verification for a M R damper", Journal of vibration and shock, vol. 33, no. 7, (2014), pp. 221-226

[5] T. Shiraishi, N. Nakaya and S. Morishita, "Structural control by a variable damper using MR fluid", The $6^{\text {th }}$ International Conference on Motion and Vibration Control, Saita-ma, Japan, vol. 6, no. 1, (2002), pp. 24-29.

[6] Y. Jia, Y. Wu and G. Zhang, "An Experimental Systemfor Semi-Active Control of Landing Gear", Research and exploration in laboratory, vol. 25, no. 2, (2006), pp. 173-175.

[7] Y. Jia and X. Wu, "Fuzzy control oflanding gear based on M R damper", Journal of Beijing / University of Aeronautics and Astronautics, vol. 33, no. 11, (2007), pp. 1264-1267.

[8] H. Liu, H. Gu and D. Wu, "Shock-absorber performance study of semi-active control of landing gear", Acta Aeronautica et Astronautica Sinica, vol. 27, no. 5, (2006), pp. 866-868.

[9] W. Wang and P. Xia, "Adaptive Control of Helicopter Ground Resonance with Magnetorheological Damper" Chinese Journal of Aeronautics, vol. 20, (2007), pp. 501-510

[10] J. Tian, L.Ding and L.-S. Kong, "Simulation and Analysis of Semi-Active Control for MR Damper Landing Gear", Chinese Hydraulics \& Pneumatics, vol. 2, (2012), pp. 47-50

[11] J. Ou, "Vibration control of structure-active, semi-active and intelligent control", Beijing: Science Press, (2003).

[12] H.-S. Hu, "The kinetics of Magnetorheological damper under̂ impact load Modeling and Sliding model control", Chinese Journal of Mechanical Engineering, (2011) vol. 47, no. 13, pp. 84-91.

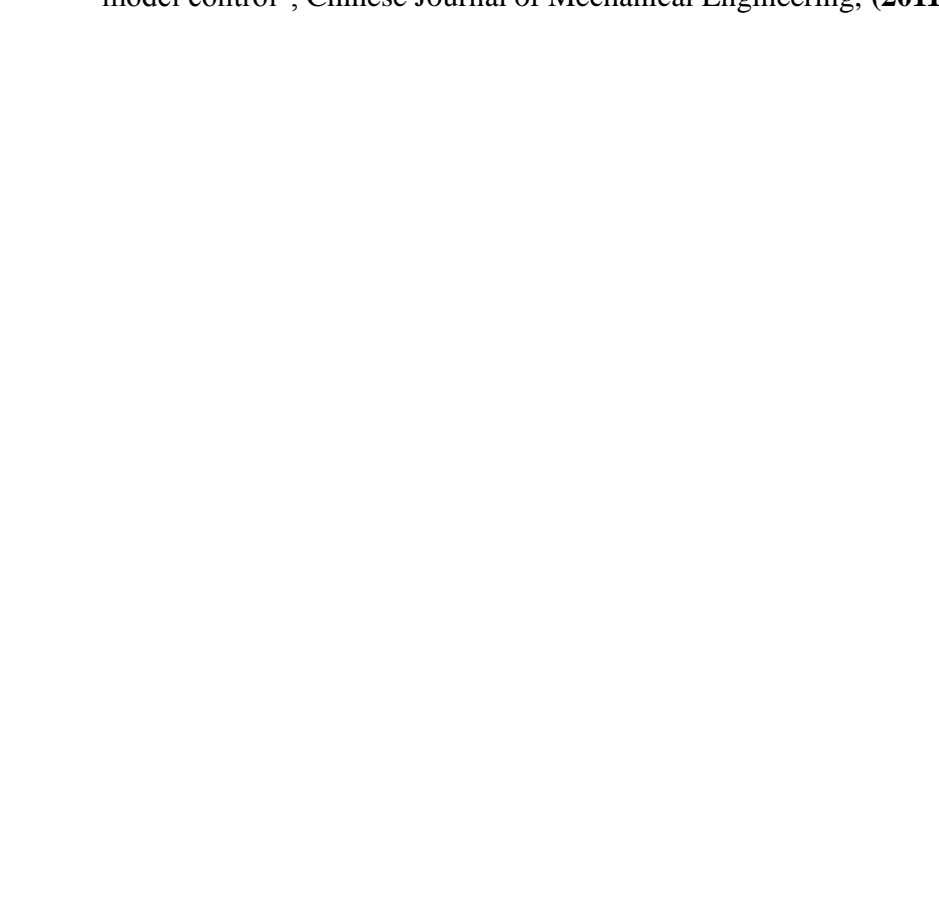


International Journal of Hybrid Information Technology

Vol. 9, No.9 (2016)

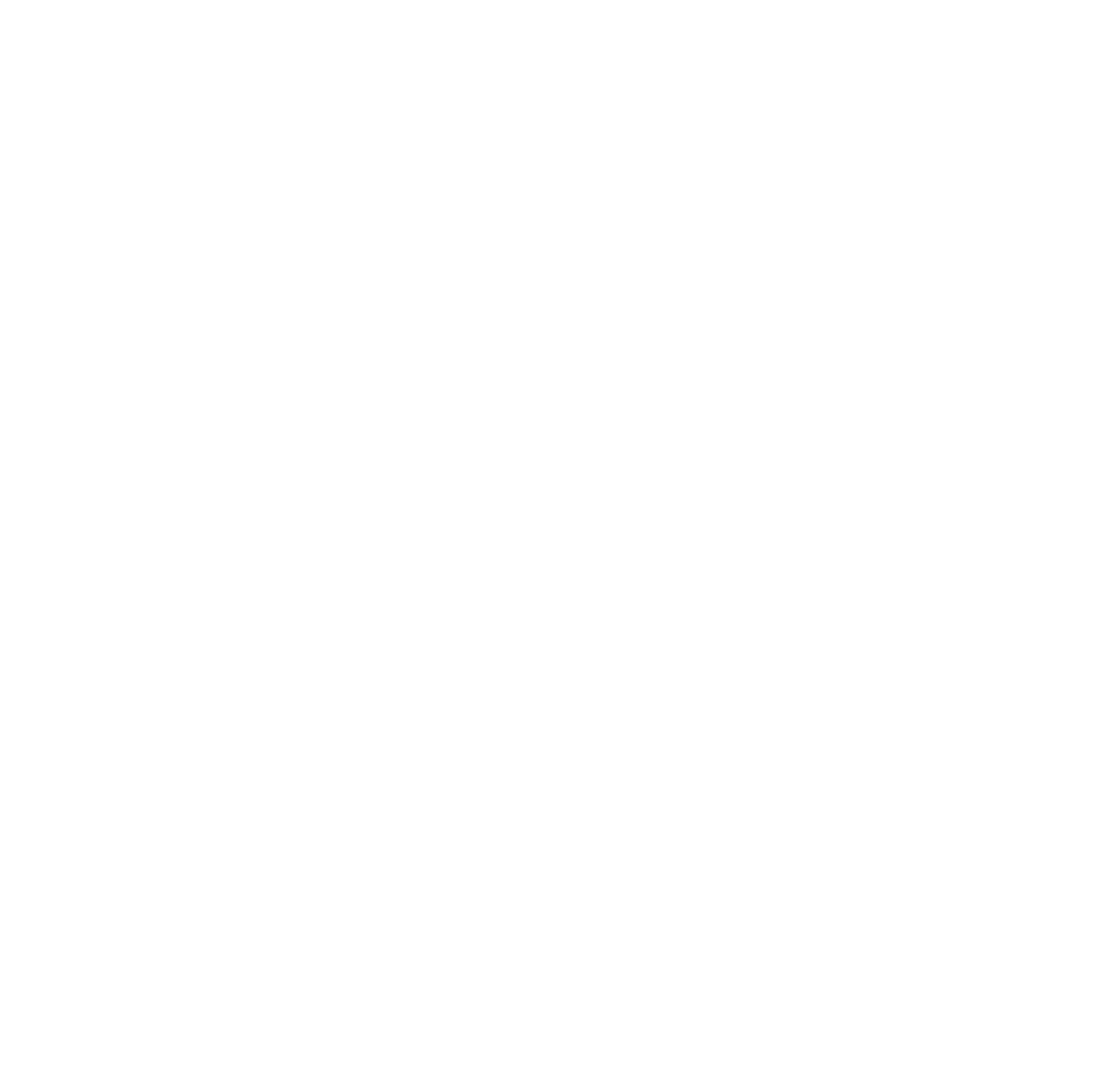

